\title{
Influential Article Review - Physical Response to Transition and Organizational Wellbeing
}

\author{
Nellie Carson \\ Carwyn Weston
}

Keri Love

This paper examines corporate social responsibility. We present insights from a highly influential paper. Here are the highlights from this paper: Organizational change has become inevitable. Although modernity is characterized by change, humans struggle with change as the future becomes increasingly unpredictable. The extended disorder that has become the norm can cause a sense of loss as well as anxiety for individuals, organizations and society.

Unrelieved stress becomes perpetual and will undeniably influence organizational change efforts negatively. Thus, the impact of fear, anxiety and stress at an individual and organizational level is accentuated.

This study followed a qualitative research approach and adopted a grounded theory methodology. Negative emotions dominated the research data which further indicated that individuals find it difficult to engage with change in a meaningful manner as fear, anxiety and stress dominate.

Thus, human reactions to change are discussed, as the organizational challenge seems to be how to find workable methods to reduce fear, anxiety and stress. Else it may become persistent, intense, chronic or recurring. Real-life stresses may then further hamper individual functioning.

The researcher conceptualizes the anti-leader and anti-manager. These concepts depict the negative characteristics of leadership and management which invariably increase individuals' fear, anxiety and stress levels. Emotions elicited by the anti-leader or anti-manager could potentially split, divide and fragment a workforce. For our overseas readers, we then present the insights from this paper in Spanish, French, Portuguese, and German.

Keywords: coherence, human reaction to change, leadership, organizational change, stress

\section{SUMMARY}

- The research data revealed that individuals were particularly stressed by organisational change, to such an extent that many participants were unable to function optimally, disconnected from the task 
at hand or were unable to think of ways to improve their situation. The data reported feelings of uncertainty, ambiguity, indecisiveness, anxiety and stress.

- Participants reported increased fear, anxiety and stress levels, uneasiness and feelings of worthlessness.

- Given the predominant negative statements and emotions, the research conceptualised the antileader and anti-manager.

- These concepts depict the negative characteristics of leadership and management.

- Enormous change is required in South Africa and Africa at large, in organisations and teams and at an individual level.

- This requires leadership commitment towards and awareness of the individual change process. The individual change process entails personality indicators coupled with the reality that fear and survival are prominent emotions during change.

- Leadership characteristics such as greed, power and inefficiency featured prominently.

- Management should encompass basic elements such as planning, organising, directing and control. During various interviews in this study, the antithesis of management was exposed. Once again following the logic of Taleb, this led the researcher to develop the concept of the anti-manager or anti-management. The concept of anti-management does not exist in the current literature, and as such, the researcher conceptualised this as a new, important key term in the field of organisational change and leadership.

- Conformity not only creates stability but also poses a real threat to organisational survival. Organisational change dents conformity, but during organisational change, anxiety becomes stable and permanent. The fear individuals experience during organisational change is often without a reference point, Organisations and leaders alike are able to mitigate such fear, anxiety and stress through increasing towards responses and decreasing away responses.

- The researcher developed Figure 2, by applying the research findings on human reactions to change and stress and human niches, combined with literature on the brain.

- Figure 2, a balanced individual is able to produce balanced thoughts. An overload of away responses increases fear, anxiety and stress. Pearce reminds us that anxiety is contagious. This is displayed through the loop of anxiety, memory, new truth , ambiguity, fear and sadness only to return to anxiety. Organisational change rests on a seesaw, one end held up by a triangle of meaning, predictability and order.

\section{HIGHLY INFLUENTIAL ARTICLE}

We used the following article as a basis of our evaluation:

Blom, T. (2018). Organisational wellness: Human reaction to change. South African Journal of Business Management, 49(1).

This is the link to the publisher's website: https://sajbm.org/index.php/sajbm/article/view/2

\section{INTRODUCTION}

Change occurs on multiple concurrent paths for both the individual and the organisation. No or little respite may result in distrust, uncertainty and the reality of organisational change fatigue (Lewis, Romanaggi \& Chapple 2010). Yesterday's practices and assumptions no longer work; therefore, the purpose of organisational change is to adapt to the environment, improve performance and change employees' behavioural patterns (Leana \& Barry 2000). Change is the new normality, yet change 
implementation remains risky with low success rates as organisations struggle with effective change implementation (Armenakis \& Harris 2009).

\section{CONCLUSION}

\section{Individual Implications}

The reality of change and the resultant adaptation that is required beckon organisations to no longer ignore the impact of stress on individual functioning. Change is undoubtedly required at an individual level. However, stressed, fearful and anxious individuals find it difficult to engage with change in a meaningful manner. As indicated in Figure 2, coherence at an individual level can only be achieved when taking into account and integrating individual's mental, spiritual, physical and emotional worlds.

Alternative intervention technologies are needed which allows individual unconscious to come into consciousness; thereafter, changed perceptions and changed thoughts become possible. Emotions play a crucial role in creating meaning. The respondents in this study admitted to feelings of being overwhelmed. Alternative intervention technologies could be applied to provide a refuge from which such feelings could be reintegrated.

\section{Leadership Implications}

Individuals, leaders and disequilibrium are the foundation of organisational change fundamentals. Organisational change is chaotic, messy, unpredictable, disordered and disorganised. Resistance, costs, profit, emotions, survival, trust, ethics, involvement, communication, strategy, teams, preparation, planning, forecasting, stress, inclusivity and the changing environment are some of the dynamic elements relating to organisational change.

Given the context of organisational change, leaders should be a portal: providing a means of entrance, a doorway, for the individual to engage with change and reach eventual vicissitude. Leadership during change is pivotal to organisational and individual change. The amount of time leaders spend on change is a direct function of the change outcome. Leadership that allows the individual new ways to share, engage, understand and operate, affording individuals the opportunity to excel, prosper and exhilarate, facilitates the individual change process.

Today's leadership requires that we recognise and harness the power vested in individuals. This requires openness to different worldviews, new suggestions, diverse insights and acceptance of alternative requirements. Transparency in terms of resource allocation, information sharing, knowledge and tools could ensure the required autonomy to complete tasks. New leadership is inclusive of all, shares information and knowledge and creates an environment conducive to change.

Organisations and leaders may mitigate fear, anxiety and stress through increasing towards responses and decreasing away responses. Leaders required in the new organisation have to lead the way to re-engage the conversation between body, mind and soul. Coherence can come about when organisations engage the physical, emotional, spiritual and mental elements of the individual. Individuals need meaning. Creating individual meaning in the change process has the potential to unleash huge energy.

\section{Future Research}

Many difficulties emerged related to the appointment of leaders versus managers. Given the importance of leadership for the future sustainability of organisations, future research would be required to establish a workable solution for promotion to leadership levels as opposed to managerial positions. Closely related to the above is the concept of the anti-leader and the anti-manager. Future research could endeavour to establish the critical, detrimental characteristics of antileaders and anti-managers, while establishing approaches and techniques to mitigate their detrimental actions. 


\section{APPENDIX}

TABLE 1

DESCRIPTION OF RESEARCH STEPS AND METHODOLOGY

\begin{tabular}{|c|c|c|c|c|c|c|}
\hline Research step & Purpose of research step & Data collect method & Data analysis & Industry & Ensuring quality data & Research question examples \\
\hline Solicited data & Provides research context & Case study & Content analysis & Motor Manufacturing & \multirow{5}{*}{$\begin{array}{l}\text { Multi-model method of data } \\
\text { collection; } \\
\text { Multiple sources of data gathering; } \\
\text { Data triangulation; } \\
\text { Recognised the multifaceted, } \\
\text { multidimensionality relating to the } \\
\text { topic; } \\
\text { Controlling definitions were used } \\
\text { throughout; } \\
\text { Constant comparison of incidents } \\
\text { to concepts; } \\
\text { Constantly fitted and refitted } \\
\text { categories. }\end{array}$} & $\cdot$ \\
\hline $\begin{array}{l}\text { Three senior } \\
\text { (regional) leaders }\end{array}$ & $\begin{array}{l}\text { To understand the role of leadership } \\
\text { and the concept of organisational } \\
\text { change from a strategy perspective }\end{array}$ & In-depth interviews & $\begin{array}{l}\text { Content analysis, descriptive } \\
\text { statistics, coding of themes } \\
\text { (grounded theory) }\end{array}$ & $\begin{array}{l}\text { Technology, Hospitality and } \\
\text { Leadership training }\end{array}$ & & \multirow{4}{*}{$\begin{array}{l}\text { Are you aware of change models? If, so which are } \\
\text { influential? } \\
\text { What are the forces driving change? } \\
\text { What types of change have you been exposed to? } \\
\text { How does your organisation prepare for change? } \\
\text { Critical success factors? } \\
\text { Do you effectively translate the business vision } \\
\text { into a change vision? } \\
\text { How important is leadership in the change } \\
\text { process? } \\
\text { How do employees perceive change? }\end{array}$} \\
\hline $\begin{array}{l}\text { Two ex-CEOs } \\
\text { (important } \\
\text { shareholders) }\end{array}$ & . & . & . & $\begin{array}{l}\text { Chemical (Rubber and } \\
\text { Fertiliser) }\end{array}$ & & \\
\hline Two CEOS & - & . & - & $\begin{array}{l}\text { Chemical (Food Ingredients } \\
\text { and Alcohol) }\end{array}$ & & \\
\hline One divisional head & - & - & - & Chemical (Fertiliser) & & \\
\hline Senior managers & $\begin{array}{l}\text { To understand the impact of } \\
\text { organisational change initiatives on } \\
\text { individuals and leaders }\end{array}$ & Focus groups & $\begin{array}{l}\text { Content analysis, descriptive } \\
\text { statistics, coding of themes } \\
\text { (grounded theory) }\end{array}$ & Manufacturing & - & \multirow{2}{*}{$\begin{array}{l}\text { Have you been part of change? } \\
\text { Was the change explained? } \\
\text { How did the change make you feel? } \\
\text { Was help pavailable? } \\
\text { Did you fully participate? } \\
\text { Did the change have a s shedule? } \\
\text { What was the role of leadership? } \\
\text { Which are critical success factors? } \\
\text { Which factors are detrimental to change? }\end{array}$} \\
\hline Middle managers & $\begin{array}{l}\text { To explore the impact of } \\
\text { organisational change on middle } \\
\text { management and understand their } \\
\text { role in implementation }\end{array}$ & - & - & Manufacturing & - & \\
\hline $\begin{array}{l}\text { Minutes, project } \\
\text { work }\end{array}$ & $\begin{array}{l}\text { To contextualise the impact of } \\
\text { organisational change }\end{array}$ & Solicited data & Content analysis & Manufacturing & - & . \\
\hline Personal notes & $\begin{array}{l}\text { To contextualise the impact of } \\
\text { organisational change }\end{array}$ & Field notes & - & Across all above industries & - & - \\
\hline $\begin{array}{l}\text { Behavioural and } \\
\text { non-behavioural }\end{array}$ & $\begin{array}{l}\text { To understand engagement and } \\
\text { change reactions }\end{array}$ & Observations & . & - & - & . \\
\hline
\end{tabular}

TABLE 2

CASE STUDY THEMES EXAMPLE

\begin{tabular}{|c|c|c|c|}
\hline Themes & Change 1 & Change 2 & Change 2 \\
\hline Participation and inclusivity & (2) & : & :) \\
\hline Communication, feedback, discussion & (2) & : & $\oplus$ \\
\hline Leadership & (2) & : & 2) \\
\hline Organisational competency to handle change & (2) & : & (2) \\
\hline Management & 8 & : & : \\
\hline Stress, anxiety and fear & (2) & : & (2) \\
\hline Resistance & (2) & (2) & (2) \\
\hline
\end{tabular}


FIGURE 1

BASIC ELEMENTS OF THE WHOLE PERSON

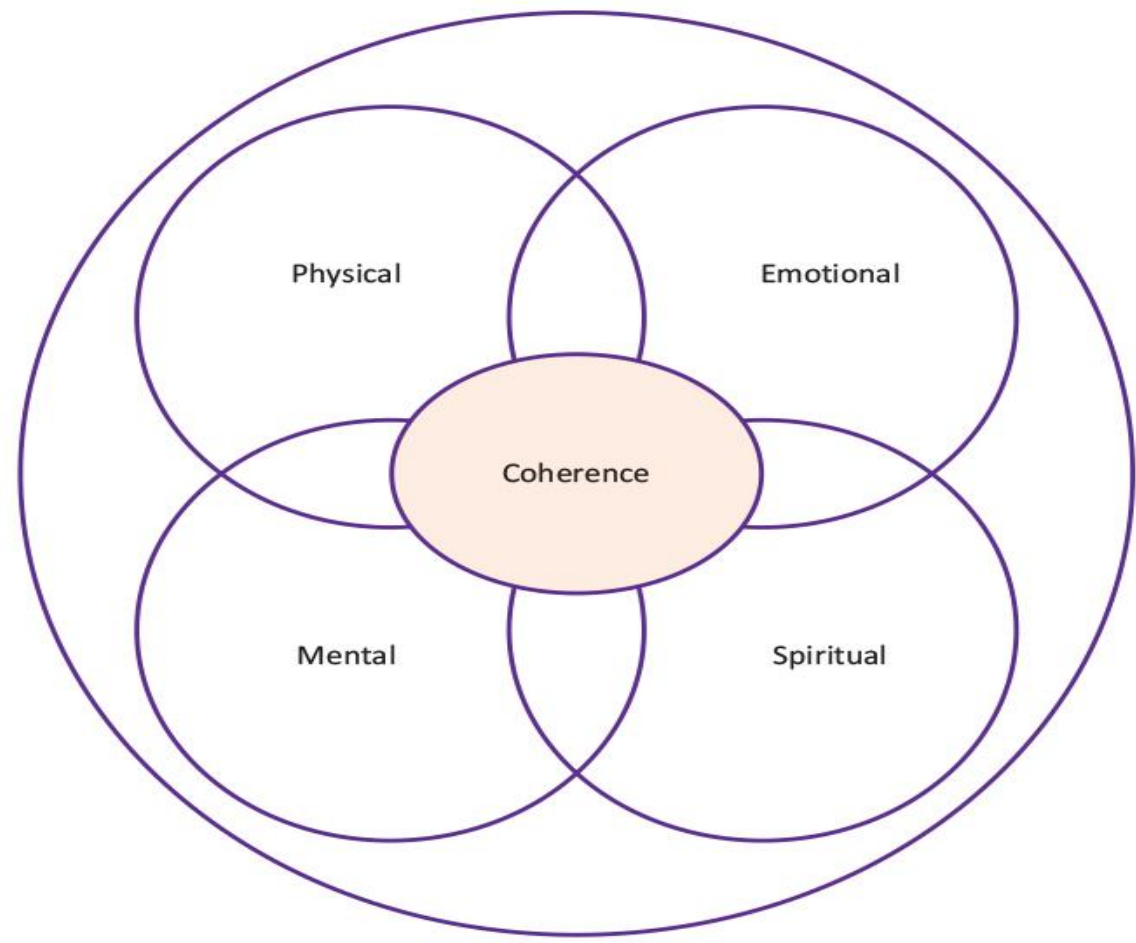

FIGURE 2

STRESS AND THE INDIVIDUAL

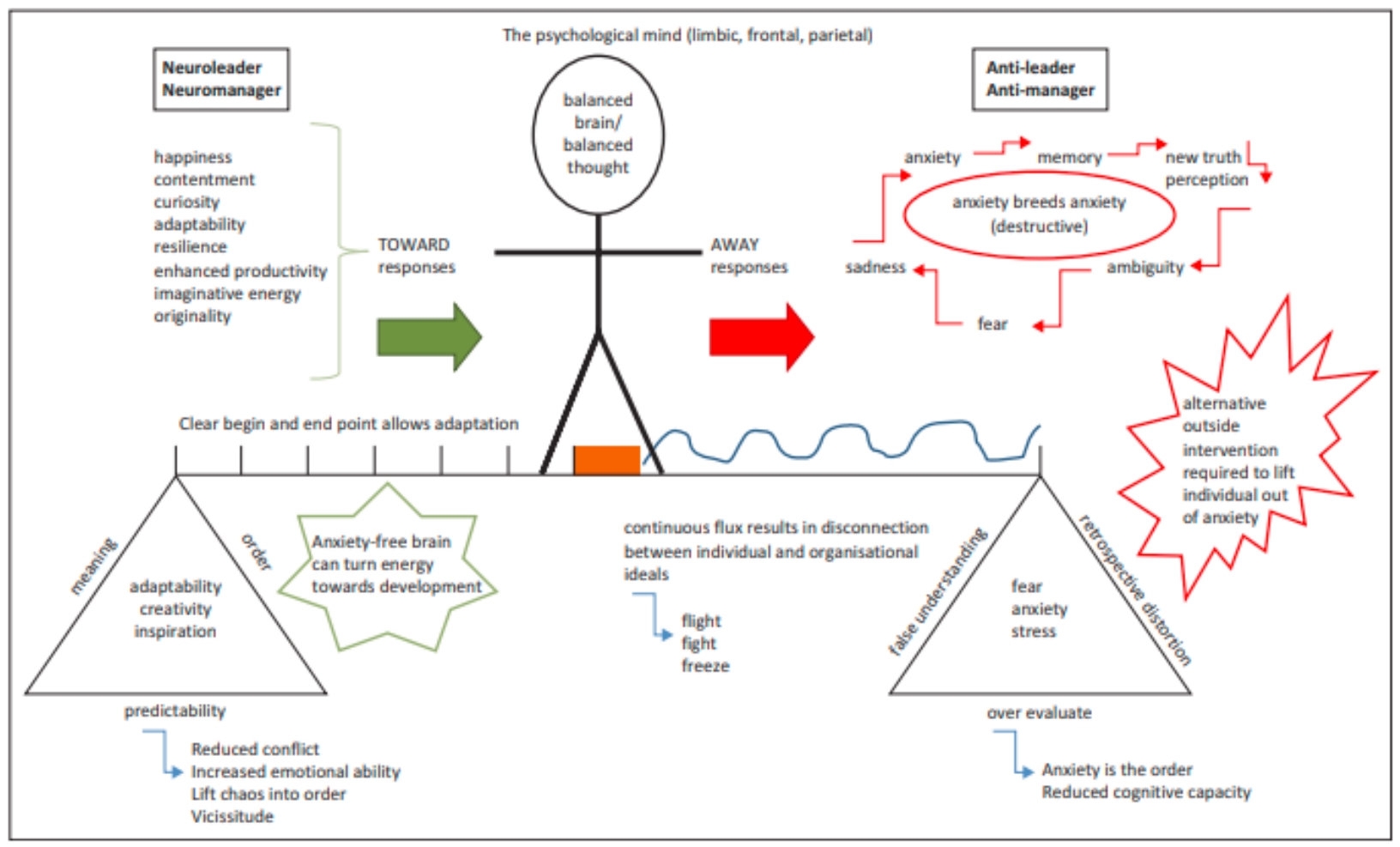


FIGURE 3

HE INDIVIDUAL'S AWAY RESPONSES AS REACTIONS TO CHANGE

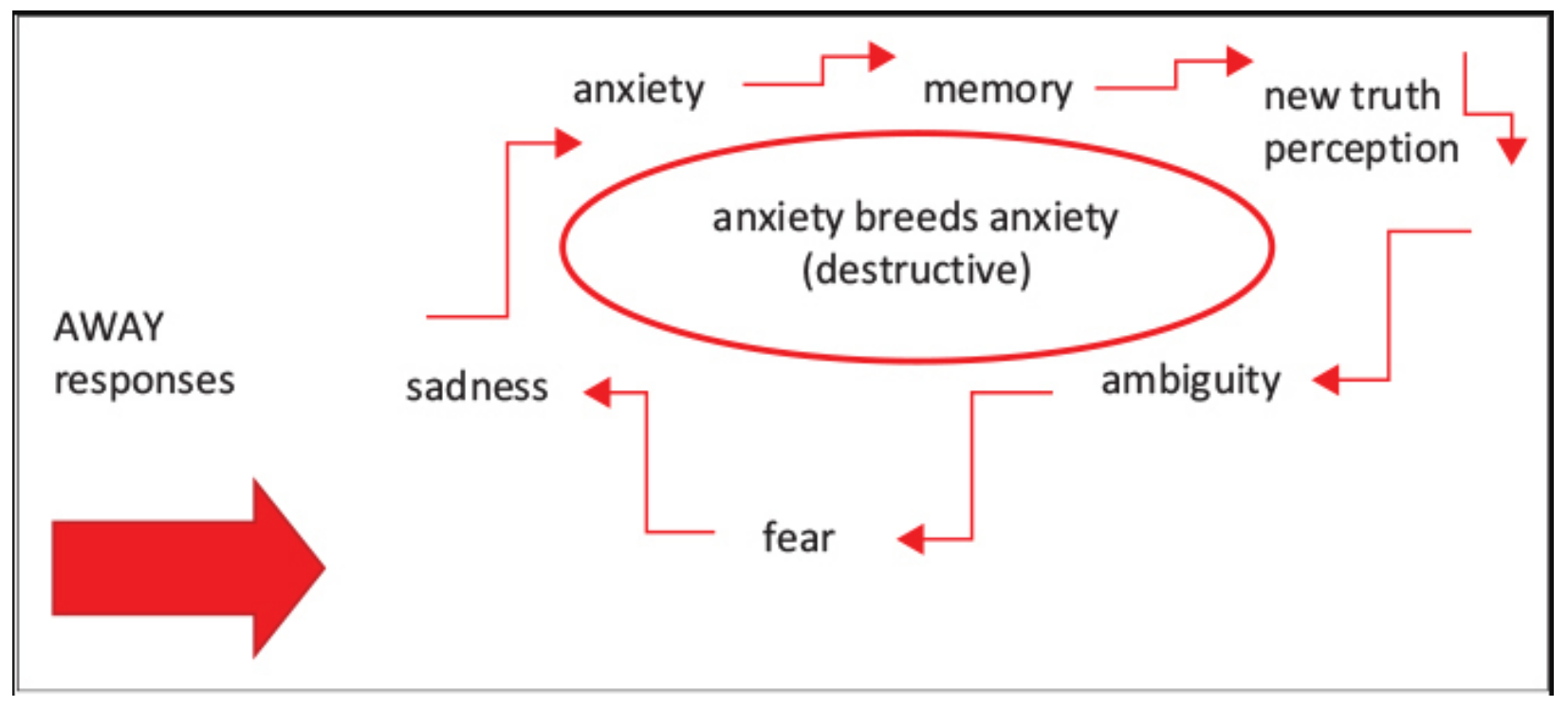

\section{REFERENCES}

Armenakis, A., \& Harris, S. (2009). Reflections: Our journey in organisational change research and practice. Journal of Change Management, 92, 127-142. https://doi.org/10.1080/14697010902879079

Berceli, D. (2012). The revolutionary trauma release process: Transcend your toughest times (5th ed.) Namaste Publishing, Vancouver.

Byrne, R. (2006, November 1). He thinks different. Business Week, p.20.

Cohen, S. (2004). Social relationships and health. American Psychologist, 59, 676-684. https://doi.org/10.1037/0003-066X.59.8.676

Darling, J.R., \& Heller, V.L. (2011). The key for effective stress management: Importance of responsive leadership in organisational development. Organisational Development Journal, 291, 1-26.

Dickerson, S.S., \& Kennedy, M.E. (2004). Acute stressors and cortisol responses: A theoretical integration and synthesis of laboratory research. Psychological Bulletin, 1303, 355-391. https://doi.org/10.1037/0033-2909.130.3.355

Dukemedicine Healthnews. (2014). Can stress cause cognitive decline? I'm in a deadline-driven, pressure-cooker job, and I'm beginning to be nervous about the effects of stress on my wellbeing. Duke Medicine Health News, 20(7), 8. ISSN: 2153-8387, PMID: 25029741, MEDLINE.

Goldsmith, M., \& Reiter, M. (2007). What got you here won't get you there. Hyperion, New York.

Goleman, D. (1995). Emotional intelligence: Why it can matter more than IQ. Bantam Books, New York.

Goleman, D. (2006). Social intelligence: The new science of human relationships. Bantam Books, New York.

Grady, J., \& Grady, V. (2011). 'Organisational mistrust: Exploring the issues, pondering its fate ...' Organisational and Social Dynamics, 11(1), 41-58.

Graves, C.W. (1978). 'Up the existential staircase', in Seminar on the development, nature, meaning and management of the levels of existence, emergent, cyclical, double helix model of adult human psychosocial coping systems, [1978/same paper with graphs added 1980, seminar in Dallas], 1974. Seminar sponsored by the National Values Center, Washington, DC.

Hannan, M.T., \& Freeman, J. (1989). Organizational ecology. Harvard University Press, Cambridge. 
Hannan, M.T., Baron, J.N., Hsu, G., \& Koğcak, Ö. (2006). Organizational identities and the hazard of change. Industrial and Corporate Change, 15, 755-784. https://doi.org/10.1093/icc/dt1020

Harung, H., Travis, F., Blank, W., \& Heaton, D. (2009). Higher development, brain integration, and excellence in leadership. Management Decision, 47(6), 872-894. https://doi.org/10.1108/00251740910966631

Isabella, L. (1990). Evolving interpretations as a change unfolds: How managers construe key organizational events. Academy of Management Journal, 33, 7-41. https://doi.org/10.2307/256350

Jones, L., Watson, B., Hobman, E., Bordia, P., Gallois, C., \& Callan, V.J. (2008). Employee perceptions of organizational change: Impact of hierarchical level. Leadership and Organisation Development Journal, 294, 294-316. https://doi.org/10.1108/01437730810876122

Kleisch, E., Sloan, A., \& Melvin, E. (2017). Using a Faculty Training and Development Model to Prepare Faculty to Facilitate an Adaptive Learning Online Classroom Designed for Adult Learners. Journal of Higher Education Theory and Practice, 17(7). Retrieved from https://articlegateway.com/index.php/JHETP/article/view/1470

Kusnecov, A., \& Rabin, B.S. (1992). Stressor-induced alterations of immune function: Mechanisms and issues. International Archives of Allergy and Immunology, 105, 108.

Lazarus, R.S. (1991). Emotion and adaptation. Oxford University Press, New York.

Leana, C.R., \& Barry, B. (2000). Stability and change as simultaneous experiences in organisational life. Academy of Management Review, 254, 753-759.

LeDoux, J. (1996). The emotional brain: The mysterious underpinnings of emotional life. Simon \& Schuster, New York.

LeDoux, J. (2002). Synaptic self: How our brains become who we are. Viking, New York.

Lewis, E., Romanaggi, D., \& Chapple, A. (2010). Successfully managing change during uncertain times. Strategic HR Review, 92, 12-18. https://doi.org/10.1108/14754391011022217

Maté, G. (2010). In the realm of hungry ghosts: Close encounters with addiction. North Atlantic Books, Berkeley, CA.

McDonald, P. (2013). The potential contribution of neuroscience to authentic leadership. In D. Rock \& A. Ringleb (Eds.), Neuroleadership (pp. 263-285). NeuroLeadership Institute ${ }^{\mathrm{TM}}$, Printed in the USA. ISBN-10:1483925331

Morris, W.N., Worchel, S., Bois, J.L., Pearson, J.A., Rountree, C.A., Samaha, G.M., . . Wright, S.L. (1976). Collective coping with stress: Group reactions to fear, anxiety, and ambiguity. Journal of Personality and Social Psychology, 33(6), 674-679. https://doi.org/10.1037/0022-3514.33.6.674

O’Connor, M., Cooper, J.N., Williams, L.M., DeVarney, S., \& Gordon, E. (2013). Neuroleadership and the productive brain. In D. Rock \& A. Ringleb (Eds.), Neuroleadership (p.481). NeuroLeadership Institute $^{\mathrm{TM}}$, Printed in the USA. ISBN-10:1483925331

Ochsner, K. (2013). Staying cool under pressure: Insights from social-cognitive neuroscience and their implications for self and society. In D. Rock \& A. Ringleb (Eds.), Neuroleadership (pp. 193204). NeuroLeadership Institute ${ }^{\mathrm{TM}}$, Printed in the USA. ISBN-10:1483925331

Pearce, J.C. (2003). Spiritual initiation and the breakthrough of consciousness: The bond of power. Park Stress Press, Rochester, VT.

Psychology Dictionary. (n.d.). Psychology Dictionary. Retrieved November 12, 2015, from http://psychologydictionary.org/pressure/

Ray, O. (2004). How the mind hurts and heals the body. American Psychologist, 591, 29-40. https://doi.org/10.1037/0003-066X.59.1.29

Sapolsky, R.M. (2004). Why Zebras don't get ulcers. 175 Fifth Avenue, St Martins Press, New York.

Schabracq, M., \& Cooper, C. (1998). Toward a phenomenological framework for the study of work and organizational stress. Human Relations, 51, 625-648.

https://doi.org/10.1177/001872679805100503

Selye, H. (1956). The stress of life. McGraw-Hill, New York.

Selye, H. (1964). From dream to discovery. McGraw-Hill, New York. 
Selye, H. (1980). The stress concept today. In I.L. Kutash, L.B. Schlesinger, \& Associates

(Eds.), Handbook on stress anxiety (pp. 127-143). Jossey-Bass, San Francisco, CA.

Selye, H. (1987). Stress without distress. Transworld, London.

Sullivan, R.M., \& Gratton, A. (2002). Prefrontal cortical regulation of hypothalamic-pituitary-adrenal function in the rat and implications for psychopathology: Side matters.

Psychoneuroendocrinology, 27, 99-114. https://doi.org/10.1016/S0306-4530(01)00038-5

Swanepoel, B., Erasmus, B., van Wyk, M., \& Schenk, H. (2003). South African human resource management: Theory and practice (3rd ed.). Juta, Lansdowne, South Africa.

Taleb, N.N. (2010). The black swan: The impact of the highly improbable (2nd ed.). Penguin, London. Thornton, E. (2009, January 19). Managing through a crisis: The new rules. Business Week, pp. 30-34.

Vijayraghavan, S., Wang, M., Birnbaum, S.G., Williams, G.V., \& Van Arnsten, A. (2007). Inverted-u dopamine D1 receptor actions on prefrontal neurons engaged in working memory. Nature Neuroscience, 10, 376-384. https://doi.org/10.1038/nn1846

\section{TRANSLATED VERSION: SPANISH}

Below is a rough translation of the insights presented above. This was done to give a general understanding of the ideas presented in the paper. Please excuse any grammatical mistakes and do not hold the original authors responsible for these mistakes.

\section{VERSION TRADUCIDA: ESPAÑOL}

A continuación se muestra una traducción aproximada de las ideas presentadas anteriormente. Esto se hizo para dar una comprensión general de las ideas presentadas en el documento. Por favor, disculpe cualquier error gramatical y no responsabilite a los autores originales de estos errores.

\section{INTRODUCCIÓN}

El cambio se produce en múltiples rutas simultáneas tanto para el individuo como para la organización. Ningún o poco respiro puede resultar en desconfianza, incertidumbre y la realidad de la fatiga del cambio organizacional (Lewis, Romanaggi y Chapple 2010). Las prácticas y suposiciones de ayer ya no funcionan; por lo tanto, el propósito del cambio organizacional es adaptarse al entorno, mejorar el rendimiento y cambiar los patrones de comportamiento de los empleados (Leana \& Barry 2000). El cambio es la nueva normalidad, sin embargo, la implementación del cambio sigue siendo arriesga con bajas tasas de éxito a medida que las organizaciones luchan con la implementación efectiva del cambio (Armenakis y Harris 2009).

\section{CONCLUSIÓN}

\section{Individual Implicaciones}

La realidad del cambio y la adaptación resultante que se requiere invita a las organizaciones a no ignorar más el impacto del estrés en el funcionamiento individual. Sin duda, el cambio es necesario a nivel individual. Sin embargo, a las personas estresadas, temerosas y ansiosas les resulta difícil comprometerse con el cambio de una manera significativa. Como se indica en la Figura 2, la coherencia a nivel individual solo puede lograrse teniendo en cuenta e integrando los mundos mental, espiritual, físico y emocional del individuo.

Se necesitan tecnologías de intervención alternativas que permitan que el inconsciente individual entre en conciencia; a partir de entonces, las percepciones cambiadas y los pensamientos cambiados se vuelven posibles. Las emociones juegan un papel crucial en la creación de significado. Los encuestados en este estudio admitieron sentimientos de estar abrumados. Podrían aplicarse tecnologías de intervención alternativas para proporcionar un refugio del que se pudieran reintegrar esos sentimientos. 


\section{Implicaciones del Liderazgo}

Los individuos, los líderes y el desequilibrio son la base de los fundamentos del cambio organizacional. El cambio organizacional es caótico, desordenado, impredecible, desordenado y desorganizado. Resistencia, costes, beneficios, emociones, supervivencia, confianza, ética, implicación, comunicación, estrategia, equipos, preparación, planificación, previsión, estrés, inclusión y el entorno cambiante son algunos de los elementos dinámicos relacionados con el cambio organizacional.

Dado el contexto del cambio organizacional, los líderes deben ser un portal: proporcionar un medio de entrada, una puerta, para que el individuo se involucre con el cambio y alcance una eventual vicisitude. El liderazgo durante el cambio es fundamental para el cambio organizacional e individual. La cantidad de tiempo que los líderes dedican al cambio es una función directa del resultado del cambio. El liderazgo que permite al individuo nuevas formas de compartir, involucrar, comprender y operar, brindando a los individuos la oportunidad de sobresalir, prosperar y entusiasmarse, facilita el proceso de cambio individual.

El liderazgo de hoy requiere que reconozcamos y aprovechemos el poder conferido a los individuos. Esto requiere apertura a diferentes visiones del mundo, nuevas sugerencias, ideas diversas y aceptación de requisitos alternativos. La transparencia en términos de asignación de recursos, intercambio de información, conocimientos y herramientas podría garantizar la autonomía necesaria para completar las tareas. El nuevo liderazgo es inclusivo para todos, comparte información y conocimientos y crea un entorno propicio para el cambio.

Las organizaciones y los líderes pueden mitigar el miedo, la ansiedad y el estrés a través del aumento de las respuestas y la disminución de las respuestas. Los líderes requeridos en la nueva organización tienen que liderar el camino para volver a entablar la conversación entre el cuerpo, la mente y el alma. La coherencia puede surgir cuando las organizaciones involucran los elementos físicos, emocionales, espirituales y mentales del individuo. Los individuos necesitan significado. Crear un significado individual en el proceso de cambio tiene el potencial de desatar una gran energía.

\section{Investigación Futura}

Surgieron muchas dificultades relacionadas con el nombramiento de líderes versus gerentes. Dada la importancia del liderazgo para la sostenibilidad futura de las organizaciones, se requeriría investigación futura para establecer una solución viable para la promoción a niveles de liderazgo en lugar de puestos gerenciales. Estrechamente relacionado con lo anterior está el concepto del anti-líder y el anti-gerente. La investigación futura podría tratar de establecer las características críticas y perjudiciales de los antilíderes y los anti-gerentes, al tiempo que se establecen enfoques y técnicas para mitigar sus acciones perjudiciales.

\section{TRANSLATED VERSION: FRENCH}

Below is a rough translation of the insights presented above. This was done to give a general understanding of the ideas presented in the paper. Please excuse any grammatical mistakes and do not hold the original authors responsible for these mistakes.

\section{VERSION TRADUITE: FRANÇAIS}

Voici une traduction approximative des idées présentées ci-dessus. Cela a été fait pour donner une compréhension générale des idées présentées dans le document. Veuillez excuser toutes les erreurs grammaticales et ne pas tenir les auteurs originaux responsables de ces erreurs.

\section{INTRODUCTION}

Le changement se produit sur plusieurs chemins simultanés pour l'individu et l'organisation. L'absence ou le peu de répit peut entraîner de la méfiance, de l'incertitude et la réalité de la fatigue du changement organisationnel (Lewis, Romanaggi \& Chapple 2010). Les pratiques et les hypothèses d'hier ne fonctionnent plus; par conséquent, le but du changement organisationnel est de s'adapter à l'environnement, 
d'améliorer les performances et de changer les comportements des employés (Leana \& Barry 2000). Le changement est la nouvelle normalité, mais la mise en œuvre du changement reste risquée avec de faibles taux de réussite alors que les organisations luttent pour une mise en œuvre efficace du changement (Armenakis \& Harris 2009).

\section{CONCLUSION}

\section{Mplications Individuelles I}

La réalité du changement et l'adaptation qui en résulte invitent les organisations à ne plus ignorer l'impact du stress sur le fonctionnement individuel. Le changement est sans aucun doute nécessaire au niveau individuel. Cependant, les personnes stressées, craintives et anxieuses ont du mal à s'engager dans le changement de manière significative. Comme l'indique la figure 2 , la cohérence au niveau individuel ne peut être atteinte qu'en tenant compte et en intégrant les mondes mental, spirituel, physique et émotionnel de l'individu.

Des technologies d'intervention alternatives sont nécessaires pour permettre à l'inconscient individuel d'entrer en conscience; par la suite, des perceptions et des pensées modifiées deviennent possibles. Les émotions jouent un rôle crucial dans la création de sens. Les répondants à cette étude ont admis avoir le sentiment d'être dépassés. D'autres technologies d'intervention pourraient être appliquées pour fournir un refuge à partir duquel ces sentiments pourraient être réintégrés.

\section{Leadership Implications}

Les individus, les leaders et le déséquilibre sont à la base des fondamentaux du changement organisationnel. Le changement organisationnel est chaotique, désordonné, imprévisible, désordonné et désorganisé. La résistance, les coûts, le profit, les émotions, la survie, la confiance, l'éthique, l'implication, la communication, la stratégie, les équipes, la préparation, la planification, les prévisions, le stress, l'inclusion et l'environnement changeant sont quelques-uns des éléments dynamiques liés au changement organisationnel.

Compte tenu du contexte du changement organisationnel, les leaders devraient être un portail: fournir un moyen d'entrée, une porte, pour que l'individu s'engage dans le changement et atteigne une éventuelle vicissitude. Le leadership pendant le changement est essentiel au changement organisationnel et individuel. Le temps que les dirigeants consacrent au changement est une fonction directe du résultat du changement. Le leadership qui permet à l'individu de nouvelles façons de partager, de s'engager, de comprendre et de fonctionner, offrant aux individus la possibilité d'exceller, de prospérer et d'exalter, facilite le processus de changement individuel.

Le leadership d'aujourd'hui exige que nous reconnaissions et exploitions le pouvoir dévolu aux individus. Cela nécessite une ouverture aux différentes visions du monde, de nouvelles suggestions, des idées diverses et l'acceptation d'exigences alternatives. La transparence en termes d'allocation des ressources, de partage de l'information, de connaissances et d'outils pourrait assurer l'autonomie requise pour accomplir les tâches. Le nouveau leadership est inclusif pour tous, partage l'information et les connaissances et crée un environnement propice au changement.

Les organisations et les dirigeants peuvent atténuer la peur, l'anxiété et le stress en augmentant les réponses et en diminuant les réponses. Les leaders requis dans la nouvelle organisation doivent montrer la voie pour réengager la conversation entre le corps, l'esprit et l'âme. La cohérence peut se réaliser lorsque les organisations engagent les éléments physiques, émotionnels, spirituels et mentaux de l'individu. Les individus ont besoin de sens. Créer un sens individuel dans le processus de changement a le potentiel de libérer une énergie énorme.

\section{Recherches Futures}

De nombreuses difficultés sont apparues liées à la nomination des dirigeants par rapport aux gestionnaires. Compte tenu de l'importance du leadership pour la durabilité future des organisations, des recherches futures seraient nécessaires pour établir une solution viable pour la promotion aux niveaux de 
leadership par opposition aux postes de direction. Étroitement lié à ce qui précède est le concept de l'antileader et de l'anti-manager. Les recherches futures pourraient s'efforcer d'établir les caractéristiques critiques et préjudiciables des antileaders et des anti-gestionnaires, tout en établissant des approches et des techniques pour atténuer leurs actions préjudiciables.

\section{TRANSLATED VERSION: GERMAN}

Below is a rough translation of the insights presented above. This was done to give a general understanding of the ideas presented in the paper. Please excuse any grammatical mistakes and do not hold the original authors responsible for these mistakes.

\section{ÜBERSETZTE VERSION: DEUTSCH}

Hier ist eine ungefähre Übersetzung der oben vorgestellten Ideen. Dies wurde getan, um ein allgemeines Verständnis der in dem Dokument vorgestellten Ideen zu vermitteln. Bitte entschuldigen Sie alle grammatikalischen Fehler und machen Sie die ursprünglichen Autoren nicht für diese Fehler verantwortlich.

\section{EINLEITUNG}

Veränderungen treten auf mehreren gleichzeitigen Pfaden sowohl für den Einzelnen als auch für die Organisation auf. Keine oder wenig Atempause kann zu Misstrauen, Unsicherheit und der Realität der organisatorischen Veränderungsmüdigkeit führen (Lewis, Romanaggi \& Chapple 2010). Die Praktiken und Annahmen von gestern funktionieren nicht mehr; Daher besteht der Zweck des organisatorischen Wandels darin, sich an die Umwelt anzupassen, die Leistung zu verbessern und die Verhaltensmuster der Mitarbeiter zu ändern (Leana \& Barry 2000). Veränderung ist die neue Normalität, aber die Implementierung von Veränderungen bleibt riskant mit niedrigen Erfolgsraten, da Unternehmen mit einer effektiven Implementierung von Veränderungen zu kämpfen haben (Armenakis \& Harris 2009).

\section{SCHLUSSFOLGERUNG}

\section{Individuelle I-Störungen}

Die Realität des Wandels und die daraus resultierende Anpassung, die erforderlich ist, locken Organisationen, die Auswirkungen von Stress auf das individuelle Funktionieren nicht länger zu ignorieren. Veränderung ist zweifellos auf individueller Ebene erforderlich. Gestresste, ängstliche und ängstliche Menschen finden es jedoch schwierig, sich sinnvoll mit Veränderungen auseinanderzusetzen. Wie in Abbildung 2 dargestellt, kann Kohärenz auf individueller Ebene nur erreicht werden, wenn die mentale, spirituelle, physische und emotionale Welt des Einzelnen berücksichtigt und integriert wird.

Es werden alternative Interventionstechnologien benötigt, die es dem individuellen Unbewussten ermöglichen, ins Bewusstsein zu kommen; danach werden veränderte Wahrnehmungen und veränderte Gedanken möglich. Emotionen spielen eine entscheidende Rolle bei der Schaffung von Bedeutung. Die Befragten in dieser Studie gaben zu, überwältigt zu sein. Alternative Interventionstechnologien könnten eingesetzt werden, um eine Zuflucht zu bieten, aus der solche Gefühle wieder integriert werden könnten.

\section{Implikationen für Führungskräfte}

Individuen, Führungskräfte und Ungleichgewicht sind die Grundlage für organisatorische Veränderungsgrundlagen. Organisatorische Veränderungen sind chaotisch, chaotisch, unvorhersehbar, ungeordnet und unorganisiert. Widerstand, Kosten, Gewinn, Emotionen, Überleben, Vertrauen, Ethik, Engagement, Kommunikation, Strategie, Teams, Vorbereitung, Planung, Prognose, Stress, Inklusivität und das sich verändernde Umfeld sind einige der dynamischen Elemente im Zusammenhang mit organisatorischen Veränderungen. 
Angesichts des Kontexts des organisatorischen Wandels sollten Führungskräfte ein Portal sein: ein Zugangsmittel, eine Tür, für den Einzelnen, um sich mit Veränderungen auseinanderzusetzen und eventuelle Wechselfälle zu erreichen. Führung während des Wandels ist entscheidend für organisatorische und individuelle Veränderungen. Die Zeit, die Führungskräfte für Veränderungen aufwenden, ist eine direkte Funktion des Veränderungsergebnisses. Führung, die es dem Einzelnen ermöglicht, neue Wege zu teilen, zu engagieren, $\mathrm{zu}$ verstehen und zu betreiben, und einzelpersonen die Möglichkeit gibt, sich zu übertreffen, zu gedeihen und zu begeistern, erleichtert den individuellen Veränderungsprozess.

Die heutige Führung erfordert, dass wir die Macht des Einzelnen anerkennen und nutzen. Dies erfordert Offenheit für unterschiedliche Weltanschauungen, neue Anregungen, vielfältige Einsichten und die Akzeptanz alternativer Anforderungen. Transparenz in Bezug auf Ressourcenzuweisung, Informationsaustausch, Wissen und Werkzeuge könnte die erforderliche Autonomie bei der Erschließung von Aufgaben gewährleisten. Neue Führungskräfte sind inklusive aller, teilen Informationen und Wissen und schaffen ein Umfeld, das Veränderungen förderlich ist.

Organisationen und Führungskräfte können Angst, Angst und Stress lindern, indem sie die Reaktionen erhöhen und die Reaktionen verringern. Führungskräfte, die in der neuen Organisation benötigt werden, müssen den Weg weisen, um das Gespräch zwischen Körper, Geist und Seele wieder in Dienung zu bringen. Kohärenz kann entstehen, wenn Organisationen die physischen, emotionalen, spirituellen und mentalen Elemente des Individuums einbeziehen. Individuen brauchen Sinn. Individuelle Bedeutung im Veränderungsprozess zu schaffen, hat das Potenzial, enorme Energie freizusetzen.

\section{Zukünftige Forschung}

Viele Schwierigkeiten traten im Zusammenhang mit der Ernennung von Führungskräften im Vergleich zu Managern auf. Angesichts der Bedeutung von Führung für die zukünftige Nachhaltigkeit von Organisationen wäre zukünftige Forschung erforderlich, um eine praktikable Lösung für den Aufstieg in Führungsebenen im Gegensatz zu Führungspositionen zu finden. Eng verwandt mit dem oben Genannten ist das Konzept des Anti-Führers und des Anti-Managers. Zukünftige Forschung könnte sich bemühen, die kritischen, nachteiligen Eigenschaften von Anti-Führungskräften und Anti-Managern zu ermitteln und gleichzeitig Ansätze und Techniken zu entwickeln, um ihre nachteiligen Handlungen zu mildern.

\section{TRANSLATED VERSION: PORTUGUESE}

Below is a rough translation of the insights presented above. This was done to give a general understanding of the ideas presented in the paper. Please excuse any grammatical mistakes and do not hold the original authors responsible for these mistakes.

\section{VERSÃO TRADUZIDA: PORTUGUÊS}

Aqui está uma tradução aproximada das ideias acima apresentadas. Isto foi feito para dar uma compreensão geral das ideias apresentadas no documento. Por favor, desculpe todos os erros gramaticais e não responsacule os autores originais responsáveis por estes erros.

\section{INTRODUÇÃO}

A mudança ocorre em vários caminhos simultâneos tanto para o indivíduo quanto para a organização. Nenhuma ou pouca pausa pode resultar em desconfiança, incerteza e a realidade da fadiga da mudança organizacional (Lewis, Romanaggi \& Chapple 2010). As práticas e suposições de ontem não funcionam mais; portanto, o objetivo da mudança organizacional é adaptar-se ao ambiente, melhorar o desempenho e mudar os padrões comportamentais dos funcionários (Leana \& Barry 2000). A mudança é a nova normalidade, mas a implementação de mudanças permanece arriscada com baixas taxas de sucesso à medida que as organizações lutam com a implementação efetiva de mudanças (Armenakis \& Harris 2009). 


\section{CONCLUSÃO}

\section{Mplications Individuais I}

A realidade da mudança e a adaptação resultante que é necessária às organizações acenam para que as organizações não ignorem mais o impacto do estresse no funcionamento individual. A mudança é, sem dúvida, necessária em um nível individual. No entanto, indivíduos estressados, temerosos e ansiosos têm dificuldade em se envolver com a mudança de forma significativa. Conforme indicado na Figura 2, a coerência em nível individual só pode ser alcançada quando se leva em conta e integra os mundos mental, espiritual, físico e emocional do indivíduo.

São necessárias tecnologias alternativas de intervenção que permitam que o inconsciente individual entre na consciência; depois disso, mudanças de percepção e pensamentos alterados tornam-se possíveis. As emoções desempenham um papel crucial na criação de significado. Os entrevistados neste estudo admitiram sentimentos de sobrecarga. Tecnologias alternativas de intervenção poderiam ser aplicadas para fornecer um refúgio do qual tais sentimentos poderiam ser reintegrados.

\section{Implicações de Liderança}

Indivíduos, líderes e desequilíbrio são a base dos fundamentos da mudança organizacional. A mudança organizacional é caótica, confusa, imprevisível, desordenada e desorganizada. Resistência, custos, lucro, emoções, sobrevivência, confiança, ética, envolvimento, comunicação, estratégia, equipes, preparação, planejamento, previsão, estresse, inclusividade e o ambiente em mudança são alguns dos elementos dinâmicos relacionados à mudança organizacional.

Dado o contexto de mudança organizacional, os líderes devem ser um portal: fornecer um meio de entrada, uma porta, para que o indivíduo se envolva com a mudança e alcance eventual vicissitude. A liderança durante a mudança é fundamental para a mudança organizacional e individual. A quantidade de tempo que os líderes gastam em mudanças é uma função direta do resultado da mudança. Liderança que permite ao indivíduo novas formas de compartilhar, engajar, entender e operar, proporcionando aos indivíduos a oportunidade de se destacar, prosperar e exultar, facilita o processo de mudança individual.

A liderança de hoje exige que reconheçamos e aproveitemos o poder investido em indivíduos. Isso requer abertura para diferentes visões de mundo, novas sugestões, insights diversos e aceitação de requisitos alternativos. A transparência em termos de alocação de recursos, compartilhamento de informações, conhecimento e ferramentas poderia garantir a autonomia necessária para concluir tarefas. A nova liderança é inclusiva de todos, compartilha informações e conhecimentos e cria um ambiente propício à mudança.

Organizações e líderes podem mitigar o medo, a ansiedade e o estresse através do aumento das respostas e da diminuição das respostas. Os líderes necessários na nova organização têm que liderar o caminho para reengajar a conversa entre corpo, mente e alma. A coerência pode surgir quando as organizações envolvem os elementos físicos, emocionais, espirituais e mentais do indivíduo. Os indivíduos precisam de significado. Criar significado individual no processo de mudança tem o potencial de liberar uma enorme energia.

\section{Pesquisa Futura}

Muitas dificuldades surgiram relacionadas à nomeação de líderes versus gestores. Dada a importância da liderança para a sustentabilidade futura das organizações, futuras pesquisas seriam necessárias para estabelecer uma solução viável para a promoção aos níveis de liderança em oposição a posições gerenciais. Intimamente relacionado com o acima está o conceito do anti-líder e anti-gerente. Pesquisas futuras poderiam se esforçar para estabelecer as características críticas e prejudiciais de antileades e anti-gestores, ao mesmo tempo em que estabelecem abordagens e técnicas para mitigar suas ações prejudiciais. 